\title{
Location of secretory component on the Fc edge of dimeric IgA1 reveals insight into the role of secretory lgA1 in mucosal immunity
}

\author{
A Bonner ${ }^{1,4}$, A Almogren ${ }^{2,4}$, PB Furtado ${ }^{1}$, MA Kerr $^{3}$ and SJ Perkins ${ }^{1}$
}

\begin{abstract}
Secretory immunoglobulin A (SIgA) is the most prevalent antibody in the human body and a first line of defense in mucosal immunity. We located secretory component (SC) relative to dimeric IgA1 (dlgA1) within the SIgA1 structure using the constrained modeling of solution scattering and analytical ultracentrifugation data. The extended solution structure of dlgA1 is largely preserved within SlgA1. From conformational searches of SC locations, the best-fit SC models within SIgA1 show that SC is extended along the outermost convex edge of the Fc dimer in dlgA1. The topology of our SIgA1 structure reveals that it is able to bind to one $\mathrm{Fc} \alpha \mathrm{RI}$ receptor molecule. SC binding to the Fc dimer confers protection to SIgA1 by the masking of proteolytically susceptible surface sites from bacterial proteases in the harsh environment of the mucosa. The models support a "zipper-like" unfolding of SC upon dlgA1 in the formation and transportation of SIgA1 into the mucosa.
\end{abstract}

\section{INTRODUCTION}

The mucosal surfaces, including the gastrointestinal, respiratory, and urogenital tracts are the largest surface area in the human body in contact with the external environment. ${ }^{1-3}$ Pathogenic microorganisms, their potentially toxic products, and indeed, some components of ingested food and inhaled air are potential threats and are therefore antigenic at mucosal surfaces. Secretory immunoglobulin A (SIgA) is the principal antibody isotype present at mucosal surfaces where it mediates the first line of immune defense by neutralizing and clearing the antigens. ${ }^{1-3}$ SIgA also restricts the indigenous commensal bacterial population entering the body from the lumen by immune exclusion. More IgA is produced daily than all the other antibody classes combined. ${ }^{1} \mathrm{IgA}$ is unique in being the only immunoglobulin class that exists in multiple oligomeric states. IgA is present as two subclasses, IgA1 and IgA2, the latter of which is found in at least two allotypic forms. The heterogeneity of IgA is reflected in its presence with characteristic distributions in very different environments around the human body. ${ }^{1}$ Thus, IgA is the second most predominant antibody class in serum IgA, where it is mainly monomeric and of the IgA1 subclass. Mucosal SIgA is predominantly dimeric with approximately equal proportions of SIgA1 and SIgA2.

SIgA is produced locally in the mucosa as polymeric IgA consisting of two (or sometimes three or four) 12-domain IgA monomers, each with two Fab and one Fc regions, bound covalently through the joining $(J)$ chain at the base of the Fc structure (Figure 1). The transport of predominantly dimeric IgA ( $\operatorname{dIgA})$ across the epithelial lining into the luminal secretions, and the consequent formation of SIgA, is mediated by $\operatorname{dIg} \mathrm{A}$ binding to the polymeric immunoglobulin receptor (pIgR), which is expressed on the basolateral surface of epithelial cells. Following transcytosis of the complex across the cell and cleavage of pIgR at the luminal membrane, SIgA is released with the extracellular portion of the pIgR (now called secretory component, SC) remaining bound to the IgA dimer (Figure 1). SC consists of five immunoglobulin variable ( $V$ )-type domains (D1-D5), and contains up to seven oligosaccharide chains. SC is not only necessary for the selective transportation and correct localization and anchorage of SIgA, but it also stabilizes markedly the structure of SIgA and increases its resistance to proteolysis in the harsh environment of mucosa. ${ }^{4-7}$ In SIgA1, the SC is bound

${ }^{1}$ Division of Biosciences, Institute of Structural and Molecular Biology, University College London, London, UK. ${ }^{2}$ Department of Pathology, College of Medicine and King Khalid University Hospital, Riyadh, Saudi Arabia. ${ }^{3}$ Department of Clinical Biochemistry and Immunology, General Infirmary at Leeds, Leeds, UK. ${ }^{4}$ These authors contributed equally to this work. Correspondence: SJ Perkins (s.perkins@medsch.ucl.ac.uk) 
covalently by a disulfide bond from Cys502 to an IgA $\alpha$-chain through Cys311. On its own, SC acts as a nonspecific antibacterial agent itself, increasing the innate immunogenic capacity of SIgA to intercept and clear pathogens. ${ }^{8,9}$

Despite the major role of SIgA1 in mucosal immunity, no new information on the assembly of this complex protein has been revealed since the electron microscopy studies from 37 years ago. ${ }^{10,11}$ The molecular arrangement of the IgA1 monomers and SC within SIgA1 is as yet unknown. However, solution structures for the IgA1 monomer and dimer and free SC have been recently determined. ${ }^{12-14}$ The IgA 1 monomer has an extended and mostly T-shaped arrangement of Fab and Fc regions. ${ }^{12,15}$ In $\mathrm{dIgA} 1$, the two IgA1 monomers are orientated in a near-planar, tail-to-tail conformation with a slight bend in the Fc-Fc interface which may possibly accommodate the J chain. ${ }^{14}$ Free SC shows a compact folded-back domain arrangement of its five domains. ${ }^{13}$ The number of multiple domains constituting SIgA1, together with its high glycosylation and flexible hinge and tailpiece regions (Figure 1), suggests that the crystallization of intact SIgA1 will be difficult to achieve. However, a solution structure can be determined at medium resolution using

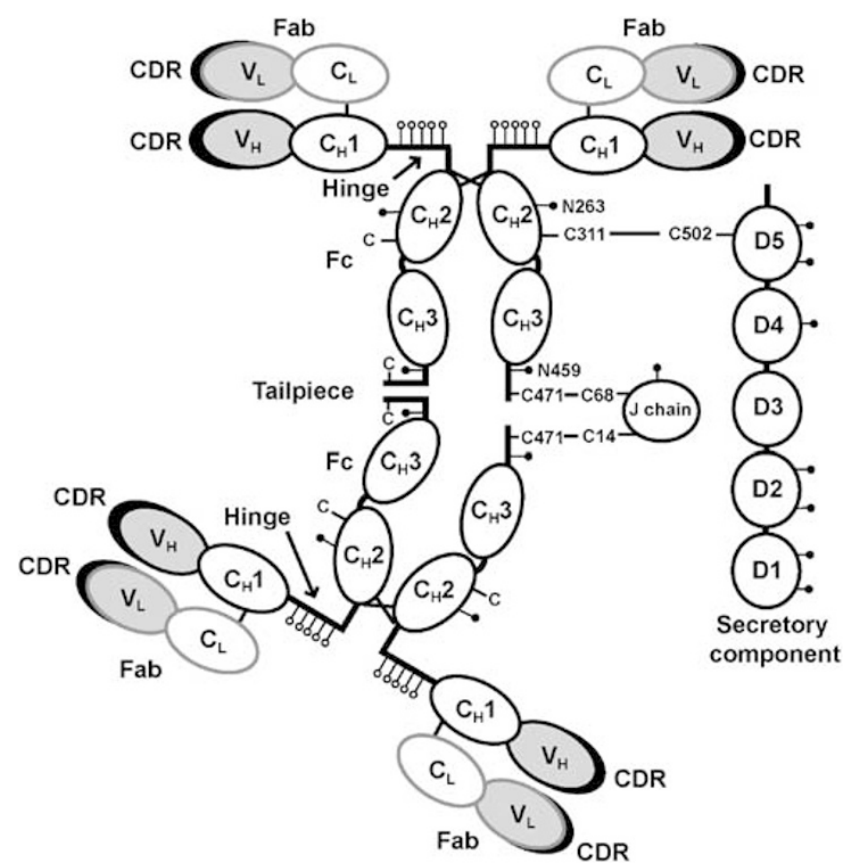

Figure 1 Domain structure of secretory immunoglobulin A1 (SIgA1). SIgA1 is composed of two IgA1 monomers that are covalently bound with the $\mathrm{J}$ chain and domains $\mathrm{D} 1$ to $\mathrm{D} 5$ of secretory component. Each IgA1 heavy chain contains the $V_{\mathrm{H}}, \mathrm{C}_{\mathrm{H}} 1, \mathrm{C}_{\mathrm{H}} 2$ and $\mathrm{C}_{\mathrm{H}} 3$ domains, and each light chain contains the $V_{\mathrm{L}}$ and $C_{\mathrm{L}}$ domains. The variable $(V)$ and constant $(C)$ are highlighted by gray and white backgrounds, respectively. The complementarity determining regions (CDR, black crescents) are indicated as labeled. The inter-heavy chain disulfide bridges at Cys241, Cys242, Cys299, and Cys301 are denoted by an extended X. Cys471 in one tailpiece of each $\mathrm{Fc}$ region is disulfide-bridged with either Cys 14 or Cys68 in the $\mathrm{J}$ chain, which is located on the convex edge of the $\mathrm{Fc}-\mathrm{Fc}$ region. Cys311 in one $\mathrm{C}_{\mathrm{H}} 2$ domain is covalently linked with Cys502 in secretory component (SC) D5. The $\mathrm{N}$-linked oligosaccharide sites at Asn263 and Asn459 are denoted by filled symbols $(\bullet)$. The five $O$-linked oligosaccharides in each hinge are denoted by open symbols $(0)$. constrained scattering modeling. ${ }^{16}$ Here, we present the first molecular view of the assembly of SIgA1. Our SIgA1 structure rationalizes its known effector functions in mucosal secretions. We discuss the implications of this SIgA1 structure in reference to its role in mucosal immunity, particularly its interaction with receptors, a mechanism for the selective transport of SIgA1 into the mucosa, and how SC and $\operatorname{dIgA} 1$ protect each other within SIgA1.

\section{RESULTS}

The same three well-defined physicochemical techniques that were employed in our other papers ${ }^{12-15}$ concerning the compo-
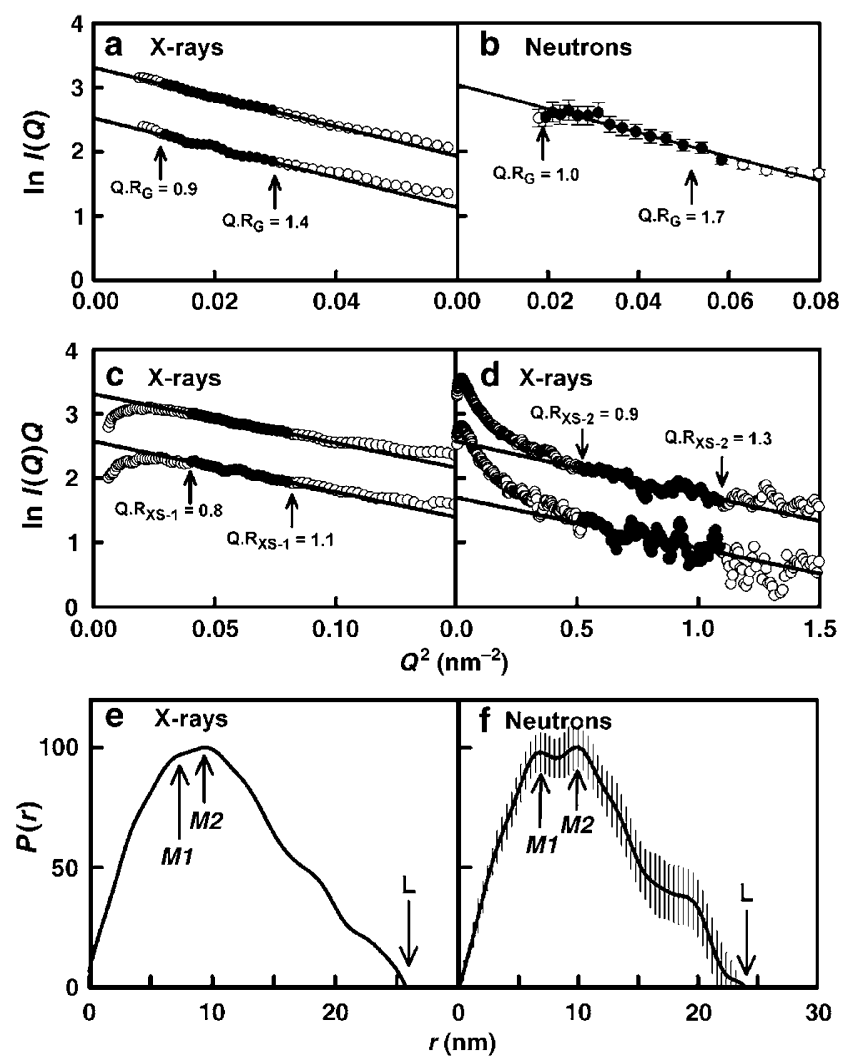

Figure 2 Guinier plots and distance distribution functions $P(r)$ analyses for secretory $\lg A 1$ (SIgA 1$)$. The scattering vector $Q$ is given by $4 \pi \sin$ $\theta / \lambda(2 \theta=$ scattering angle; $\lambda=$ wavelength). (a-d) The Guinier plots are arbitrarily displaced on the intensity axis for reason of clarity. The $Q . R_{G}$ and $Q . R_{\mathrm{XS}}$ ranges used to determine the $R_{\mathrm{G}}, R_{\mathrm{XS}-1}$, and $R_{\mathrm{XS}-2}$ values are represented by filled circles between the arrowed data points to show that they fall within satisfactory $Q . R_{\mathrm{G}}$ limits. The X-ray fits correspond to concentrations of $0.60 \mathrm{mg} / \mathrm{ml}$ (upper) and $0.30 \mathrm{mg} / \mathrm{ml}$ (lower). The neutron fit corresponds to data at $0.60 \mathrm{mg} / \mathrm{ml}$. (a, b) The

X-ray and neutron $Q$ ranges used for the $R_{G}$ values were $0.11-0.17$ and $0.14-0.24 \mathrm{~nm}^{-1}$, respectively. (c, d) The X-ray $Q$ ranges used to calculate the $R_{\mathrm{XS}-1}$ and $R_{\mathrm{XS}-2}$ values were $0.20-0.28$ and $0.72-1.04 \mathrm{~nm}^{-1}$, respectively. No neutron cross-sectional analyses were calculated for reason of insufficient signal-noise ratios. (e, $\mathbf{f})$ The two most frequently occurring distances in the $P(r)$ curve are denoted by the maxima $M 1$ and $M 2$ at 7.0 and $9.6 \mathrm{~nm}$ (X-ray) and 6.8 and $10.0 \mathrm{~nm}$, respectively (neutron). The maximum length of SIgA1 is denoted by $L$ at $26 \mathrm{~nm}$ (X-ray) and $24 \mathrm{~nm}$ (neutron) when $P(r)$ reaches 0 . The mean $R_{\mathrm{G}}$ values determined from the X-ray and neutron $P(r)$ curves of $8.30 \pm 0.17 \mathrm{~nm}$ (six values) and $8.04 \mathrm{~nm}$ (one value), respectively were in good agreement with the Guinier analyses of $\mathbf{a}$ and $\mathbf{b}$. 
nents of SIgA1 were used to obtain solution structural data on intact SIgA1. The data were then fitted to a library of conformationally randomized models to identify the spatial arrangement of the individual components within SIgA1 that best described the three data sets.

\section{X-ray and neutron scattering of SIgA1}

Solution scattering is a diffraction technique that studies the overall structure and size of biological macromolecules in random orientations. ${ }^{16} \mathrm{X}$-ray scattering showed that SIgA1 and dIgA1 have similar elongated arrangements of two IgA1 monomers. On the basis of the data at the lowest scattering angles, Guinier analyses revealed that the mean X-ray $R_{\mathrm{G}}$ value (a monitor of macromolecular elongation) for SIgA1 was $8.29 \pm 0.20 \mathrm{~nm}$ (Figure 2a,b; Table 1). This is slightly less than that for dIgA1 at $8.65 \pm 0.27 \mathrm{~nm} .{ }^{14}$ The anisotropy ratio $R_{\mathrm{G}} / R_{\mathrm{O}}$ permits the degree of elongation to be compared between different macromolecules. The ratio for SIgA1 was 1.93. This is slightly less than that of 2.16 for $\operatorname{dIgA} 1$, showing that SIgA1 is less elongated than $\operatorname{dIgA1}$. This indicated that SC ( $19 \%$ of the mass of $\operatorname{dIgA} 1)$ is bound to the central region of $\operatorname{dIgA} 1$ and not to its periphery.

Similarities in the solution structures of SIgA1 and dIgA1 were shown by cross-sectional Guinier analyses of the X-ray data at larger scattering angles. The $R_{\mathrm{XS}}$ values monitor shorter dimensions, e.g., the diameter of the Fc region. Two linear regions were revealed similar to those for $\mathrm{dIgA} 1$ that correspond to the $R_{\mathrm{XS}-1}$ and $R_{\mathrm{XS}-2}$ parameters. ${ }^{14}$ The mean $R_{\mathrm{XS}-1}$ value of $3.90 \pm 0.13 \mathrm{~nm}$ and mean $R_{\mathrm{XS}-2}$ value of $1.27 \pm 0.03 \mathrm{~nm}$ (Figure 2c,d; Table 1) were close to those for $\operatorname{dIgA} 1$ of $3.94 \pm 0.18$ and $1.43 \pm 0.07 \mathrm{~nm}$, respectively.

Neutron scattering data acted as a control of the X-ray data. Neutrons will test for any hydration effect which is much less visible by neutrons than by $\mathrm{X}$-rays, the absence of $\mathrm{X}$-ray-induced radiation damage, and any internal structural inhomogeneity caused by the $11 \%$ carbohydrate content of SIgA1. ${ }^{16}$ The neutron $R_{\mathrm{G}}$ value for SIgA1 in a heavy water buffer was $7.22 \mathrm{~nm}$ (Figure 2b), which is also less than the dIgA1 neutron value of $7.60 \mathrm{~nm}$. The reduction of $1 \mathrm{~nm}$ in the neutron $R_{\mathrm{G}}$ value for SIgA 1 compared to its $\mathrm{X}$-ray value is attributable to the masking of a significant hydration shell positioned at large distances from the center of the SIgA1 structure. The neutron $R_{\mathrm{G}} / R_{\mathrm{O}}$ ratio for SIgA1 was 1.85, which agrees well with the $\mathrm{X}$-ray value above. Neutron scattering can also be used to determine the molecular mass. The Guinier $I(0) / c$ value of 0.43 resulted in a mass of $390 \mathrm{kDa}$ for SIgA1. This compares well with that of the composition-derived value of $424 \mathrm{kDa}$, showing that SIgA1 is monodisperse in solution.

The conversion of the scattering curve $I(Q)$ into the distance distribution function $P(r)$ provides structural dimensions. The $P(r)$ curves reproducibly showed two broad maxima $M 1$ and $M 2$ located at 7.0 and $9.6 \mathrm{~nm}$ (X-ray) and 6.8 and $10.0 \mathrm{~nm}$ (neutron). Each maximum corresponds to an abundance of intermolecu-

Table 1 Summary of the X-ray and neutron modeling fits for the SgA1 solution structure

\begin{tabular}{|c|c|c|c|c|c|c|c|c|c|}
\hline $\begin{array}{l}\text { Filter for best-fit } \\
\text { models }\end{array}$ & Models & $\begin{array}{l}\text { Hydrated } \\
\text { spheres }(N)\end{array}$ & $\underset{(\mathrm{nm})}{\text { X-ray } R_{\mathrm{G}}}$ & $\begin{array}{l}\text { X-ray } \\
R_{\mathrm{XS}-1} \\
(\mathrm{~nm})\end{array}$ & $\begin{array}{c}\text { X-ray } \\
R_{\mathrm{XS}-2}(\mathrm{~nm})\end{array}$ & $\begin{array}{l}\text { Neutron } \\
R_{\mathrm{G}}(\mathrm{nm})\end{array}$ & $\begin{array}{c}\text { X-ray } R \\
\text { factor (\%) }\end{array}$ & $\begin{array}{l}\text { Neutron } \\
R \text { factor } \\
(\%)\end{array}$ & $\mathbf{S}_{20, w}^{\circ}(\mathrm{S})$ \\
\hline None & $1,500^{\mathrm{a}}$ & $4,610-5,000$ & $8.14-8.82$ & $4.03-4.88$ & $1.31-1.94$ & $7.18-7.68$ & $8.5-13.1$ & $6.8-10.7$ & NA \\
\hline $\begin{array}{l}N, R_{\mathrm{G}}, R_{\mathrm{XS}-1} \\
R \text { factor }\end{array}$ & 10 & $4,643-4,852$ & $8.25-8.37$ & $4.14-4.20$ & $1.49-1.79$ & $7.24-7.32$ & $8.5-8.8$ & $8.6-10.0$ & $11.2-11.5$ \\
\hline $\begin{array}{l}\text { Poor-fit Model } \\
1 \text { (Figure 5b) }\end{array}$ & 1 & 4,883 & 8.20 & 4.12 & 1.74 & 6.94 & 9.4 & 9.5 & 11.5 \\
\hline $\begin{array}{l}\text { Poor-fit Model } \\
2 \text { (Figure 5b) }\end{array}$ & 1 & 4,974 & 8.09 & 3.99 & 2.02 & 6.96 & 9.9 & 10.1 & 11.7 \\
\hline \multicolumn{10}{|c|}{ Randomized Fab fits } \\
\hline $\begin{array}{l}N, R_{\mathrm{G}}, R_{\mathrm{XS}-1} \\
R \text { factor }\end{array}$ & 50 & $4,680-4,797$ & $7.97-8.46$ & $3.82-4.19$ & $1.42-1.92$ & $6.82-7.07$ & $8.0-8.4$ & $9.2-10.8$ & NA \\
\hline \multicolumn{10}{|c|}{ Experimental values } \\
\hline & NA & 4,757 & $\begin{array}{l}8.29 \pm 0.20 \\
(6 \text { values })\end{array}$ & $\begin{array}{l}3.90 \pm 0.13 \\
\text { (5 values) }\end{array}$ & $\begin{array}{l}1.27 \pm 0.03 \\
(5 \text { values })\end{array}$ & 7.22 & NA & NA & $\begin{array}{c}10.8 \\
\text { (SEDFIT) } \\
10.9 \\
(\mathrm{DCDT}+) \\
\end{array}$ \\
\hline
\end{tabular}

NA, not available.

aThe 1,500 models were selected from the 5,000 starting models because they show no steric clashes between the SC domains. 
lar distances within the structure. Although two maxima were also observed for $\operatorname{dIgA1} 1{ }^{14}$ the $\mathrm{X}$-ray $M 1$ values at $6.8-7.0 \mathrm{~nm}$ for SIgA1 were larger than those of 4.9-5.1 nm for dIgA1. This movement in $M 1$ is attributed to the effect of bound SC in SIgA1. The $M 2$ values at $9.6-10.1 \mathrm{~nm}$ for SIgA1 and dIgA1 were similar. The maximum length, $L$, is determined from where the $P(r)$ curve reaches zero at large $r$. For SIgA $1, L$ was determined to be $26 \mathrm{~nm}$ (X-ray) and $24 \mathrm{~nm}$ (neutron) (Figure $2 \mathbf{e}$ and $\mathbf{f}$ ), similar to those for $\operatorname{dIg} \mathrm{A} 1$. This shows that SIgA1 and $\mathrm{dIgA} 1$ possess similar extended lengths.

\section{Analytical ultracentrifugation of SIgA1}

Analytical ultracentrifugation studies macromolecular structures in solution by following their sedimentation behavior on subjecting these to a high centrifugal force ${ }^{17}$ To confirm the molecular mass of SIgA1, sedimentation equilibrium experiments resulted in molecular masses between 360 and $420 \mathrm{kDa}$ (Figure 3). Their extrapolation to zero concentration gave a mean molecular mass of $403 \pm 43 \mathrm{kDa}$ within error of the composition-derived value of $424 \mathrm{kDa}$.

The sedimentation coefficient $s_{20, \mathrm{w}}^{\circ}$ provides an independent measure of macromolecular elongation. Good agreement between the experimental and fitted sedimentation velocity boundaries was obtained (Figure $\mathbf{4 a , c}$ ). The resulting $c(s)$ size distribution analyses revealed a single SIgA1 peak with an $s^{\circ}{ }_{20, w}$ value of $10.8 \mathrm{~S}$ (Figure $4 \mathbf{b}, \mathbf{d}$ ). The $10.8 \mathrm{~S}$ peak corresponded to a molecular mass of $440 \mathrm{kDa}$. As no other peaks were detectable, this confirmed sample monodispersity. An alternative calculation using $g\left(s^{*}\right)$ time-derivative analyses gave similar $s^{\circ}{ }_{20, \mathrm{w}}$ values of 10.8 and $10.9 \mathrm{~S}$ (Figure $4 \mathbf{e}, \mathbf{f}$ ). The new determinations agree well with a previous $s^{\circ}{ }_{20, w}$ value of $10.9 S^{18}$ and values between 10.5 and $11.7 \mathrm{~S} .{ }^{19}$ The $s^{\circ}{ }_{20, w}$ value leads to the frictional ratio $f / f_{\mathrm{o}}$, which permits comparison of the degree of elongation. The $f / f_{\mathrm{o}}$ of SIgA1 is 1.70 , which is similar to that of 1.63 for $\operatorname{dIgA} 1,{ }^{14}$ showing that SIgA 1 and $\operatorname{dIgA} 1$ have similarly elongated structures in solution.

\section{Constrained modeling of SIgA1}

Constrained modeling of the scattering and sedimentation data based on known molecular structures resulted in the SIgA1 structure. ${ }^{16}$ The presence of SC has a measurable effect on the Xray data of SIgA1 in comparison to dIgA1. When superimposed, the SIgA 1 and $\mathrm{dIgA} 1$ scattering curves differ at approximate scattering vectors $Q$ of $0.5-0.8 \mathrm{~nm}^{-1}$ (Figure 5a). Given that similar overall arrangements of the two IgA1 monomers within $\operatorname{dg} A 1$ and SIgA1 had been deduced above, the constrained modeling of SIgA1 was initiated using the best-fit dIgA1 structure. ${ }^{14}$ The first modeling searches tested 10 different locations of SC. Each search was based on the 5,000 randomized arrangements of SC domains previously used to determine the unbound SC solution structure. ${ }^{13}$ Each search positioned domain D3 or D5 of the SC models proximate to a specific part of the dIgA1 structure. For example, searches 5 and 6 tested hypothesized structures in which SC was wrapped around the planar Fc-Fc region. ${ }^{1-3,19-22}$ Searches 7-10 tested SC models based on the Cys502-Cys311 disulfide bridge (Figure 1). ${ }^{23}$
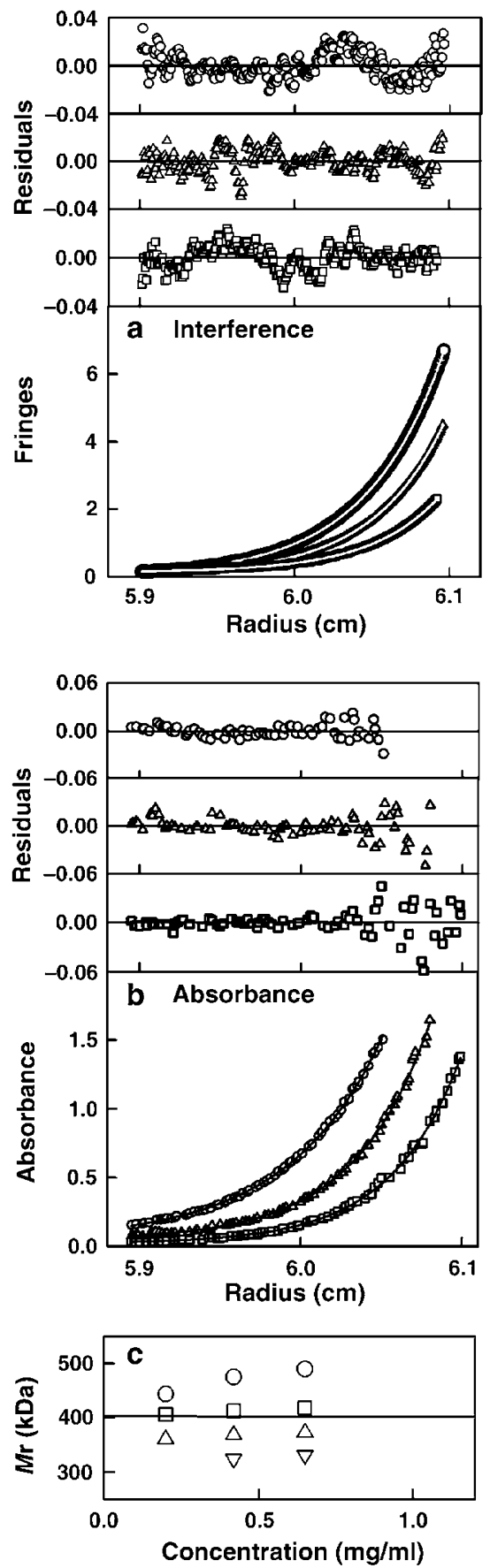

Figure 3 Sedimentation equilibrium analyses for secretory IgA1 (SlgA1). SlgA1 was analyzed using (a) interference and (b) absorbance optics at concentrations of $0.65 \mathrm{mg} / \mathrm{ml}$ (top), $0.43 \mathrm{mg} / \mathrm{ml}$ (middle), and $0.22 \mathrm{mg} / \mathrm{ml}$ (bottom) at a rotor speed of 8,000 r.p.m. Circles represent the experimental data and the continuous black lines represent their fits. The corresponding curve-fit residuals are shown above the exponential fits. (c) The rotor speed dependence of the fitted molecular mass gave a mean value of $403 \pm 10 \mathrm{kDa}$, denoted by $(\bigcirc)$ 5,000 r.p.m., ( $\square$ ) 8,000 r.p.m., $(\triangle) 11,000$ r.p.m., and $(\nabla) 14,000$ r.p.m.

The SIgA1 scattering curves from each model were calculated for comparison with the experimental data. Search 1 gave the most favorable outcome. The best-fit $R_{\mathrm{G}}$ values were close to the experimental value of $8.29 \mathrm{~nm}$ (Table 1) and gave the lowest goodness-of-fit $R$ factors, which are required for a good fit 

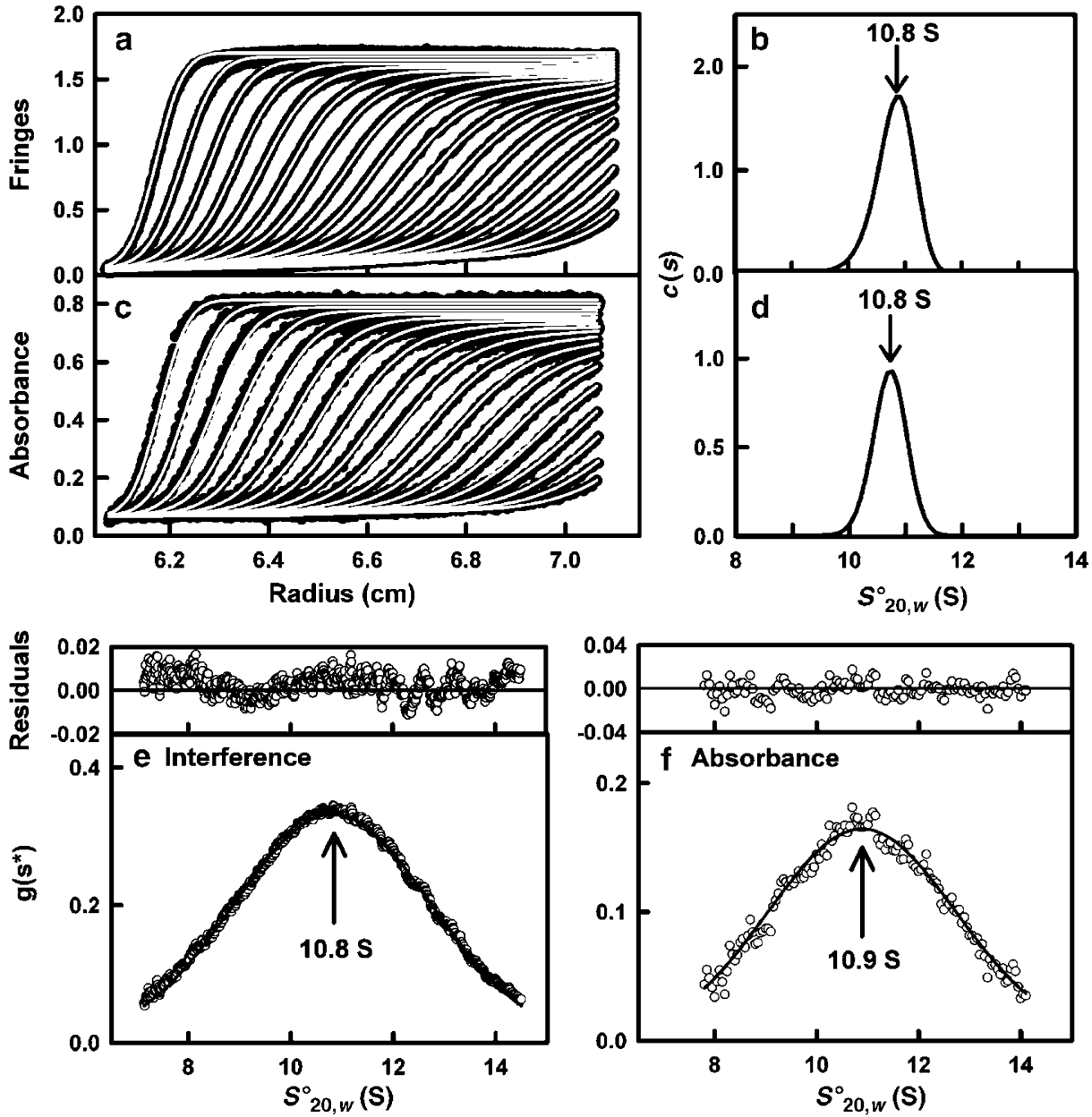

Figure 4 Sedimentation velocity analyses for secretory IgA1 (SIgA1) using SEDFIT $c(s)$ size distribution and DCDT + time-derivative fits. The black circles represent the experimental scans and the continuous white or black lines represent the fits. (a, c) In the SEDFIT fits, only every sixth scan of the 120 scans is shown for reason of clarity. Interference scans at a concentration of $0.58 \mathrm{mg} / \mathrm{ml}$ and a rotor speed of 20,000 r.p.m. are shown in (a), whereas the corresponding absorbance scans at $280 \mathrm{~nm}$ are shown in (c). The 120 scans were recorded at 5 min intervals between consecutive ones. (b, d) The corresponding $c(s)$ plots from $(\mathbf{a}, \mathbf{c})$ are shown from which the sedimentation coefficient for SIgA1 was determined to be 10.8S. (e, f) In the $\mathrm{g}\left(\mathrm{s}^{*}\right)$ fits using DCDT +, the goodness-of-fit residuals are shown above each analysis. The arrowed $s^{\circ}{ }_{20, w}$ values using (e) interference and (f) absorbance optics are 10.8 and $10.9 \mathrm{~S}$, respectively. The fits are based on data at $0.58 \mathrm{mg} / \mathrm{ml}$ (interference) and $0.58 \mathrm{mg} / \mathrm{ml}$ (absorbance) at a rotor speed of 20,000 r.p.m. The fits were determined using from 4 to 14 scans recorded mid way through the experiment shown in a and $\mathbf{c}$.

(Figure 6b). These best models also had $R_{\mathrm{XS}-1}$ and $R_{\mathrm{XS}-2}$ values close to the minimum $R$ factor (Figure $6 c$ ). Search 2 gave a similar outcome to that from search 1 (Figure 6b). The other eight searches gave worsened $R_{\mathrm{G}}$ agreements. For example, searches 3 and 4 gave reduced $R_{\mathrm{G}}$ values of $8.1-8.4 \mathrm{~nm}$ because the models were too compact, and many SC models sterically clashed with the two Fab regions. Searches 5 and 6 gave even lower $R_{\mathrm{G}}$ values of 8.0-8.2 nm. Searches 7-10 gave $R_{\mathrm{G}}$ values that were too high and no best-fit minimum in the $R$ factors could be identified.

Search 1 identified extended near-planar best-fit SC structures that were aligned along the convex edge of the $\mathrm{Fc}-\mathrm{Fc}$ plane. Their $R_{\mathrm{G}}$ and $R_{\mathrm{XS}-1}$ values were within $5 \%$ of the experimental values. The best 10 structures with the lowest $R$ factors are shown in dark gray and black in Figure 6. The best-fit SIgA1 structure has a good $R$ factor of $8.5 \%$ (black arrow in Figure 6) with a good visual X-ray curve fit in a $Q$ range extending to $1 \mathrm{~nm}^{-1}$
(Figure 5a). The corresponding neutron curve fit has a good $R$ factor of $9.3 \%$ despite a worsened signal-noise ratio. The $M$ and $L$ values of the $P(r)$ curves were well reproduced. Two poor fits from the best outcome of searches $3 / 4$ and searches $5 / 6$ illustrated the success of search 1 (Figure $5 \mathbf{b}$ ). Both poor fits showed deviations in the $Q$ range of $0.5-0.8 \mathrm{~nm}^{-1}$ that corresponded to the main difference between the $\operatorname{dIgA} 1$ and SIgA1 curves. Their $R_{\mathrm{G}}$ values were too low, and the $R$ factors were increased (Table 1).

The best-fit structure from search 1 was refined by randomization of the four Fab hinges in this SIgA1 model. The best-fit SC structure from search 1 was added to the 3,000 randomized Fab structures used previously to determine the $\operatorname{dIgA} 1$ structure. ${ }^{14}$ The best $R$ factor was reduced from 8.5 to $8.0 \%$ (Figure $7 \mathbf{a})$, and the modeled X-ray $R_{\mathrm{G}}, R_{\mathrm{XS}-1}$, and $R_{\mathrm{XS}-2}$ values were in slightly better agreement with the experimental values (Table $\mathbf{1}$ ). 


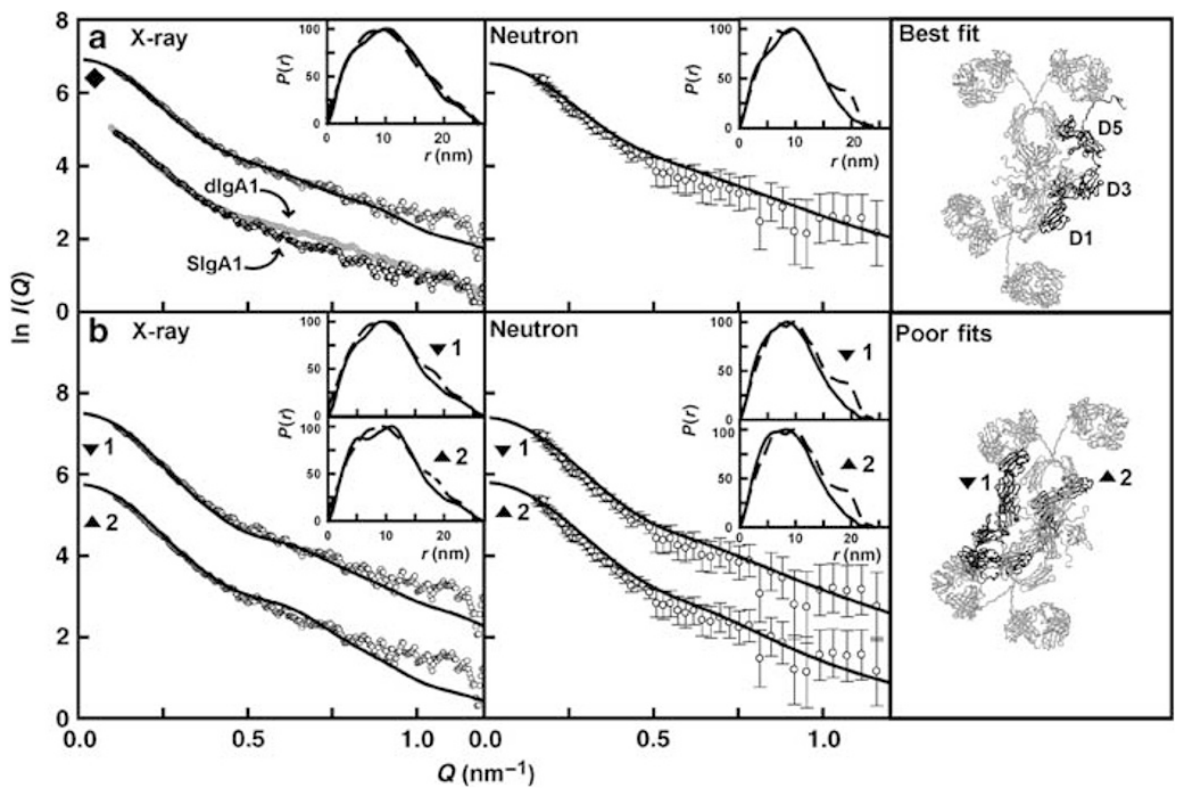

Figure 5 X-ray and neutron fits for the best-fit secretory IgA1 (SIgA1) model and two representative poor-fit SIgA1 models from search 1. (a) The X-ray and neutron experimental $I(Q)$ and $P(r)$ curves are shown as symbols $(O)$, and the modeled best-fit curves are in black. The experimental $I(Q)$ curve is shown in the main panel whereas the $P(r)$ curve (dashed) is shown as an inset in the top right corner. The comparison between the experimental X-ray $I(Q)$ curves for SlgA1 (black) and dlgA1 (gray) is also shown. The best-fit model is shown as a ribbon trace with the IgA1 dimer and $\mathrm{J}$ chain shown in gray, and domains D1 to D5 of secretory component (SC) shown in black. (b) The X-ray and neutron curve fits are shown for two poor-fit SlgA1 models 1 and 2. In model 1, SC is on the other edge of the Fc-Fc dimer compared to the best-fit SlgA1 model. In model 2, SC is positioned on the central face of the Fc-Fc dimer. Other details follow those in $\mathbf{a}$.

Superimposition of the 50 best-fit models revealed a limited conformational family of structures (Figure 7c) in which the two Fab regions displayed an extended and mostly T-shaped arrangement relative to their adjacent Fc region. All four Fab regions were independent of each other. When compared with the dIgA1 modeling, ${ }^{14}$ the Fab regions were in approximately the same positions and included many best-fit models from the dIgA1 fits. This agreement suggests that no conformational change in $\operatorname{dIgA} 1$ occurred when this binds to SC.

The experimental sedimentation coefficient $s^{\circ}{ }_{20, \mathrm{w}}$ value of $10.9 \mathrm{~S}$ for SIgA1 agreed well with the $s^{\circ}{ }_{20, \mathrm{w}}$ values of $11.2-11.5 \mathrm{~S}$ calculated from the 10 best-fit models from search 1 (Table 1). The best-fit SIgA1 model had an $s^{\circ}{ }_{20, w}$ values of 11.4 S. Slightly $\operatorname{larger} s_{20, \mathrm{w}}^{\circ}$ values of 11.5 and $11.7 \mathrm{~S}$ were obtained with the two poor-fit models of Figure 5b. These agreements support the $\mathrm{X}$-ray scattering best-fit SIgA1 model of Figure 5a.

\section{DISCUSSION}

SIgA is one of the most important components of the immune system. We have produced a solution structure at medium resolution showing how SIgA1 is assembled from $\operatorname{dIgA1}$ and SC. Comparison of the solution structure of SIgA1 and the previously reported structure of serum $\operatorname{dIgA} 1$ shows that $\operatorname{dIgA} 1$ is almost unaffected by its incorporation into SIgA1. This is consistent with published electron micrographs but might not have been expected from the much emphasized stability of SIgA1 to proteolysis and the lack of effector functions suggested in some studies. The major difference between $\mathrm{SIgA} 1$ and $\operatorname{dIgA1}$ is the novel location of SC to the outer edge of the central Fc-Fc region in SIgA1. This new knowledge enhances our understanding of SIgA1 structure and function. Thus, this structure reveals that SC is positioned to carry out its unique biological functions without altering the fundamental structure and function of the dIgA1 moiety. Our structure is based on a homogeneous SIgA1 preparation, previously demonstrated by gel filtration and sodium dodecyl sulfate-polyacrylamide gel electrophoresis, ${ }^{7}$ and confirmed here by analytical ultracentrifugation data that rule out the possibility of contamination by monomeric IgA1 or other immunoglobulin forms. The same SIgA1 preparation binds to FcaR1 (CD89), triggering a respiratory burst in neutrophils in the same way as dIgA1 from serum that lacks SC. ${ }^{24}$ This SIgA1 also binds to jacalin and is cleaved by proteases acting at both the hinge region and the $\mathrm{C}_{\mathrm{H}} 2 / \mathrm{C}_{\mathrm{H}} 3$ interface, although the latter site is cleaved at a much slower rate than serum IgA1. These results emphasize the functional accessibility of much of the SIgA1 molecule, even though SC is bound to it.

The size of SIgA1, its high carbohydrate content, and its conformational flexibility meant that at present constrained scattering modeling offered the only route to a macromolecular structure. General structural similarities between SIgA1 and dIgA1 were identified. This meant that overall SIgA1 has a nearplanar solution structure similar to that of $\operatorname{dgA} 1$, with the two IgA1 monomers orientated end-to-end through a slightly bent arrangement of their Fc regions (Figure 8a). The location of the SC structure along the outermost convex edge of the Fc-Fc region shows that SC must unfold from its compact J-shaped structure in solution. ${ }^{13}$ No conformational alteration of dIgA1 was detectable after SC is bound (Figure 7). A similar structure 

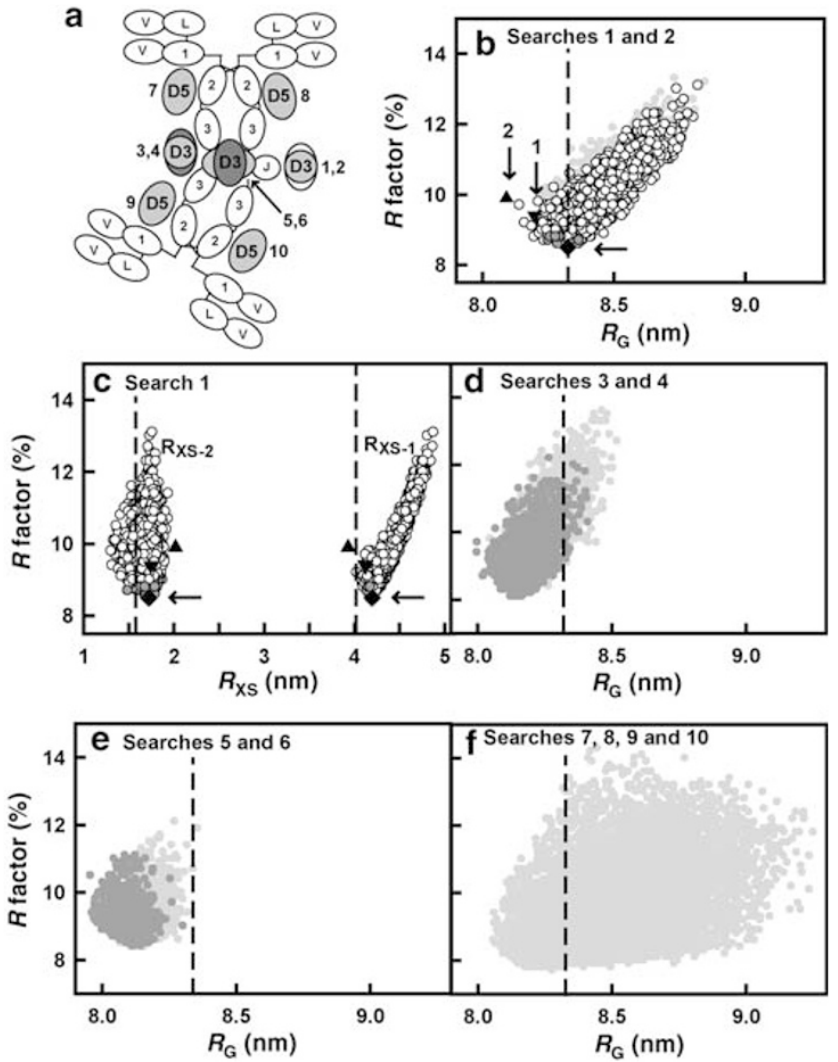

Figure 6 Constrained modeling analyses of secretory $\lg A 1$ (SIgA1). (a) The 10 searches numbered from 1 to 10 are denoted by shaded circles labeled D3 or D5 to correspond to the secretory component (SC) domain held fixed relative to dlgA1 in each search. The shaded circles are shown in relation to Figure 1. Searches 1-4 positioned D3 on the convex and concave edges of the dimeric Fc region. Search 1 held the long axis of $\mathrm{D} 3$ parallel to the long axis of $\mathrm{Fc}-\mathrm{Fc}$ and next to the putative $\mathrm{J}$ chain position on the convex edge of $\mathrm{Fc}-\mathrm{Fc}$. Search 2 repeated this with D3 now rotated by $90^{\circ}$ to be perpendicular in position to that used in search 1 . Searches 3 and 4 repeated these searches on the other concave edge of the Fc-Fc region. Searches 5 and 6 explored locations on the planar face of Fc-Fc. These positioned the long axis of D3 either parallel or perpendicular to the long axis of Fc-Fc. Searches 7-10 explored SC models based on the disulfide bridge between Cys502 in $\mathrm{D} 5$ and Cys311 in one of the four $\mathrm{C}_{\mathrm{H}^{2}}$ domains (Figure 1). Accordingly, D5 was positioned parallel to and next to each of the four $\mathrm{C}_{\mathrm{H}} 2$ domains in SlgA1, and Cys502 was located within $2.0 \mathrm{~nm}$ of Cys311. (b, c) The $R_{\mathrm{G}}, R_{\mathrm{XS}-1}$, and $R_{\mathrm{XS}-2}$ values calculated from the models in search 1 are compared with the $R$ factor values from their curve fits (Table 1). Search 2 (light gray) is shown also in (b). The vertical dashed lines correspond to the experimental $R_{\mathrm{G}}, R_{\mathrm{XS}-1}$, and $R_{\mathrm{XS}-2}$ values. The 10 best-fit models are in dark gray and black, and the best-fit model is shown as the black diamond (horizontal arrow). Two representative poor-fit models 1 and 2 are shown as black triangles (vertical arrow) (Figure 5b). (d-f) The $R_{G}$ values calculated from the models in each of searches 3 (light gray), 4 (dark gray), 5 (light gray), 6 (dark gray), and 7-10 (gray) are compared with the $R$ factor values from their curve fits.

for SIgA2 has been determined (A.B. and S.J.P., submitted for publication). Because this SIgA1 structure is an experimental structural determination, the 10 best-fit models qualify for deposition in the Protein Data Bank (Supplementary material online). These 10 structures are best viewed when superimposed to appreciate the precision of the curve-fit method (Figure 8a).
It is stressed that the molecular interactions between the five individual $\mathrm{SC}$ domains and the $\mathrm{Fc}-\mathrm{Fc}$ region in $\mathrm{dIgA} 1$ are not defined by this work, as our approach is not a high-resolution structure determination. Interestingly, our best-fit SIgA1 models resemble crystal structures for the Fc $\alpha$ RI-Fc $\alpha, F_{c} R n-F c \gamma$, Fc $\gamma$ RIII-Fc $\gamma$, and FceRI-Fce complexes (PDB codes 1ow0, $1 \mathrm{a} 1 \mathrm{i}, 1 \mathrm{e} 4 \mathrm{k}, 1 \mathrm{f} 6 \mathrm{a})^{25}$ in the sense that all four complexes show the receptor molecule making various contacts at the edge of the $\mathrm{Fc}$ region in the three antibody classes, and not at its face. The location of SC in SIgA1 also resembles the edge-to-edge contacts formed between the five $\mathrm{Fc}$ regions in the human IgM pentamer (PDB code $2 \mathrm{rcj}),{ }^{26}$ and likewise the location of protein $\mathrm{A}$ on the edge of the $\mathrm{Fc}$ region (PDB code $1 \mathrm{fc} 2)^{27}$

Scattering modeling is not able to determine unique molecular structures, however it is able to rank models in the order of best fit (Table 1). In this way, search 1 located SC on the more exposed convex edge of $\mathrm{Fc}-\mathrm{Fc}$ in SIgA1 in preference to the outcome of the nine other searches (Figure 5a). The generally accepted electron microscopy model for SIgA1 indicated an endto-end arrangement of two IgA1 monomers with various degrees of bending between them. ${ }^{11}$ This is supported by the scattering analysis. Even though no electron microscopy study located SC relative to dIgA1, the old SIgA images inspired many subsequent cartoons for SC bound to one face of dIgA1 or wrapped around $\mathrm{Fc}-\mathrm{Fc}$. The ranking of such models ruled them out of consideration (Figure 6).

Our new SIgA1 structure provides novel insights on its binding to IgA receptors. A near-planar SIgA1 structure is able to interact with cell-surface receptors, most notably Fc $\alpha$ RI, which is responsible for IgA-mediated phagocytosis, oxidative burst, and antibody-dependent cellular cytotoxicity among other roles. ${ }^{28-30}$ Receptor binding will occur independently of antigen binding as the $\mathrm{Fc}$ and Fab regions are well separated in our structure. On the basis of crystallographic analysis of the interaction of monomeric IgA and Fc $\alpha$ RI, four Fc $\alpha$ RI molecules will potentially bind to $\operatorname{dIgA} 1$, one at each junction between the $\mathrm{C}_{\mathrm{H}} 2$ and $\mathrm{C}_{\mathrm{H}} 3$ domains. ${ }^{14,31,32}$ Our location of $\mathrm{SC}$ on the outermost convex edge of the $\mathrm{Fc}-\mathrm{Fc}$ region in SIgA1 indicates that two of these four FcaRI sites will be sterically blocked by SC (Figure 8c). Our previous analysis of dIgA1 binding to Fc $\alpha$ RI suggested that only two of the four sites (specifically those on diagonally opposite $\mathrm{C}_{\mathrm{H}} 3$ domains) can bind to FcaRI at the cell surface for reason of the stereochemical orientation of $\mathrm{Fc} \alpha \mathrm{RI}$ relative to $\mathrm{Fc}$ (molecules 1 and 4 in Figure 9d of Bonner et al. ${ }^{14}$ ). As one of these two accessible FcaRI sites will be blocked by SC in SIgA1, it is expected that only one FcaRI binding site on the concave edge of the Fc region is available, leading to a 1:1 stoichiometry as shown in Figure 8c. It is predicted that both SIgA1 and dIgA1 will bind to FcaRI on cell surfaces, in accordance with experiment. ${ }^{24}$

Our SIgA1 structure clarifies the formation of SIgA1 from dIgA1 and SC during pIgR transcytosis. In dIgA1, the convex edge of the $\mathrm{Fc}-\mathrm{Fc}$ region is presumed to be the location for the $\mathrm{J}$ chain within the crevice that is created by this bend, even though the J chain could not be located by scattering modelling. ${ }^{14}$ This is consistent with the immunologically hidden nature of the J chain in $\operatorname{dIgA} 1 .{ }^{20}$ The binding of SC to the convex Fc-Fc edge 
in SIgA1 is consistent with the mandatory presence of J chain for SC binding. ${ }^{33,34}$ Unbound SC shows a J-shaped arrangement of domains in solution. ${ }^{13}$ Our SIgA1 structure shows that SC has to unfold on binding to $\operatorname{dIgA} 1$, and this is consistent with the different conformational epitopes found in free and bound SC. ${ }^{35}$ The extensive interactions between SC and the Fc-Fc edge in SIgA1 are consistent with biochemical studies. Thus, its D1 domain is important for SC to interact with dIgA1, most notably at the $\mathrm{CDR}$ regions in $\mathrm{D} 1$, but $\mathrm{D} 1$ will not bind alone to $\mathrm{Fc}^{36,37}$ The D2 and D3 domains increase the affinity for $\operatorname{dIgA} 1 .{ }^{38}$ The D5 domain interacts with Cys311 at the edge of the IgA C $\alpha 2$ domain. ${ }^{12,23}$ Accordingly our SIgA1 structure enables us to visualize a "zipper-like" unfolding of the J-shaped SC domains when this binds to $\operatorname{dgAA} 1 ., 14$ Sterically, the convex Fc edge of dIgA1 is relatively accessible in the $\mathrm{Fc}-\mathrm{Fc}$ region, and the location of SC on this edge in our model clarifies how pIgR is able to interact with $\operatorname{dIgA} 1$ to mediate transcytosis.

The SC location in SIgA1 clarifies the greater ability of SIgA1 to resist proteolysis in the harsh environment of mucosal surfaces. Unbound SC is readily degraded to half its size, ${ }^{13,20,39}$ but this is reduced in SIgA1. Trypsin, pepsin, and two bacterial proteases cleave monomeric IgA 1 and $\operatorname{dIgA} 1$ more readily than SIgA $1 .{ }^{5,6} \mathrm{SC}$ delays cleavage in the hinge/Fc region of the $\alpha$-chain. ${ }^{40}$ Potential cleavage sites are expected to occur at protein surface loops. Such sites along the convex edge of the Fc-Fc region will be blocked by SC binding in our SIgA1 structure (Figure 8). Decreased cleavage rates at the concave edge of the $\mathrm{Fc}-\mathrm{Fc}$ region in SIgA1 may result from the reduced access to proteases caused by the presence of the Fab regions. Accessibility to proteases at this edge will also be hindered by the binding of SIgA1 to cell-surface receptors. Comparison of crystal structures for two bacterial metalloproteases in Figure 8b supports this interpretation, as both are comparatively large enzymes. The active sites of the proteases are such that they will accept the substrate in only one configuration. Hence, it is not only the size of the protease but the necessity for a specific orientation relative to IgA that is relevant, as both factors will leave reduced opportunities for their active sites to access their targets. The decreased susceptibility to proteolysis may result also from reduced flexibility between the $\mathrm{Fc}$ and Fab regions after SIgA1 is formed. The binding of SC along the Fc-Fc edge of dIgA1 will reduce movements between the two IgA1 monomers, hence reducing opportunities for proteolytic attack. Further protection is expected from the surface location of eight glycosylation sites at Asn263 and Asn459 on the two Fc regions, together with seven glycosylation sites in SC (Figure 8b), ${ }^{12,13}$ that will reduce the accessibility of SIgA1 to proteases.

The elucidation of a near-planar solution structure for SIgA1 with SC positioned on the convex edge of the Fc-Fc region transforms our understanding of the function of SIgA. This opens the way for further experimentation to refine and extend our molecular understanding of SIgA assembly, and the way that SIgA1 performs its functions in mucosal immunity.

\section{METHODS}

\section{Preparation and composition of SIgA1}

Native SIgA1 containing $\lambda$ - and $\kappa$-class light chains was isolated from human colostrum provided by consenting healthy volunteers, and
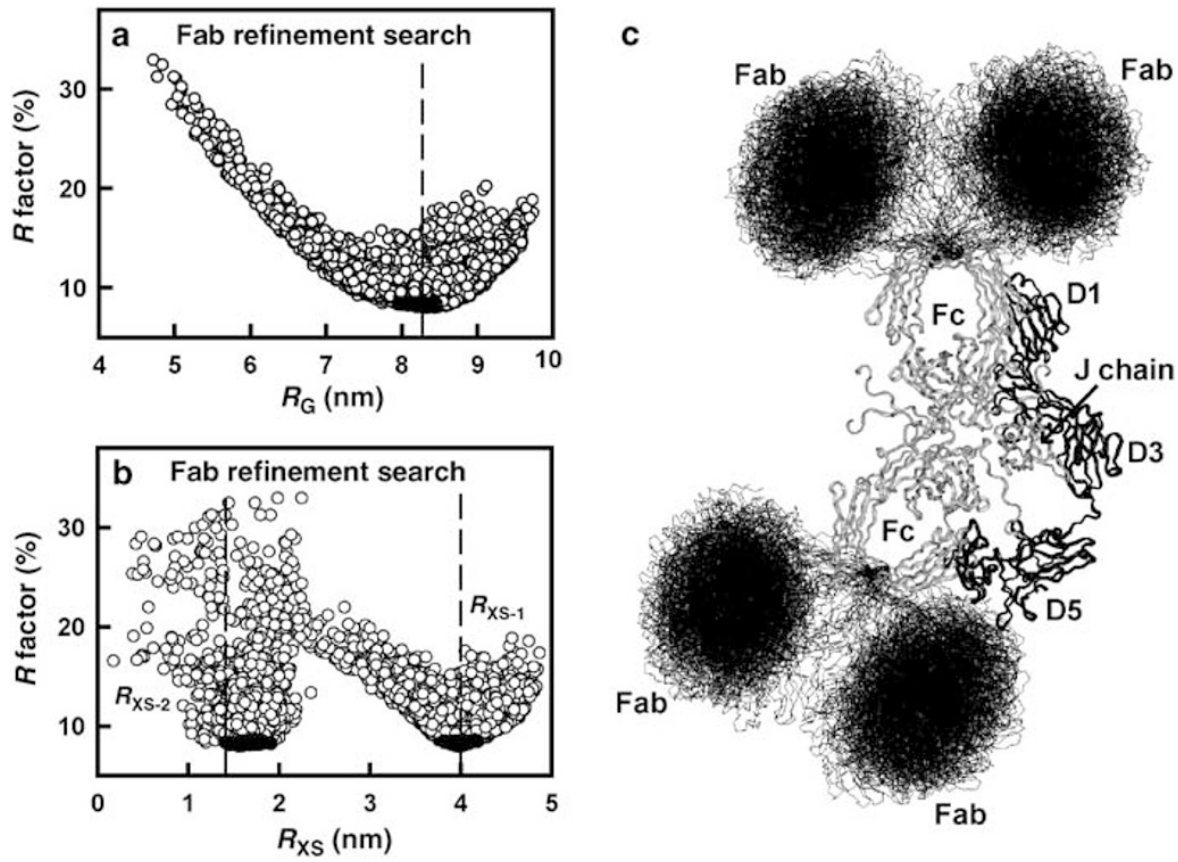

Figure 7 The best-fit models for secretory $\operatorname{lgA} 1$ (SIgA1) from a search of 3,000 randomized Fab positions. (a, b) Comparison of the X-ray $R$ factors with the X-ray $R_{\mathrm{G}}$ values for 1,500 models. The 50 best-fit models are shown in black (arrowed). The vertical dashed lines indicate the experimental X-ray $R_{\mathrm{G}}, R_{\mathrm{XS}-1}$, and $R_{\mathrm{XS}-2}$ values. (c) The superimposition of the 50 best-fit models for SIgA1, in which the Fab regions are shown as black lines, the two Fc regions are shown as gray ribbons at the center, and secretory component (SC) is shown as black ribbons with the positions of D1, D3, and D5 indicated as shown. 


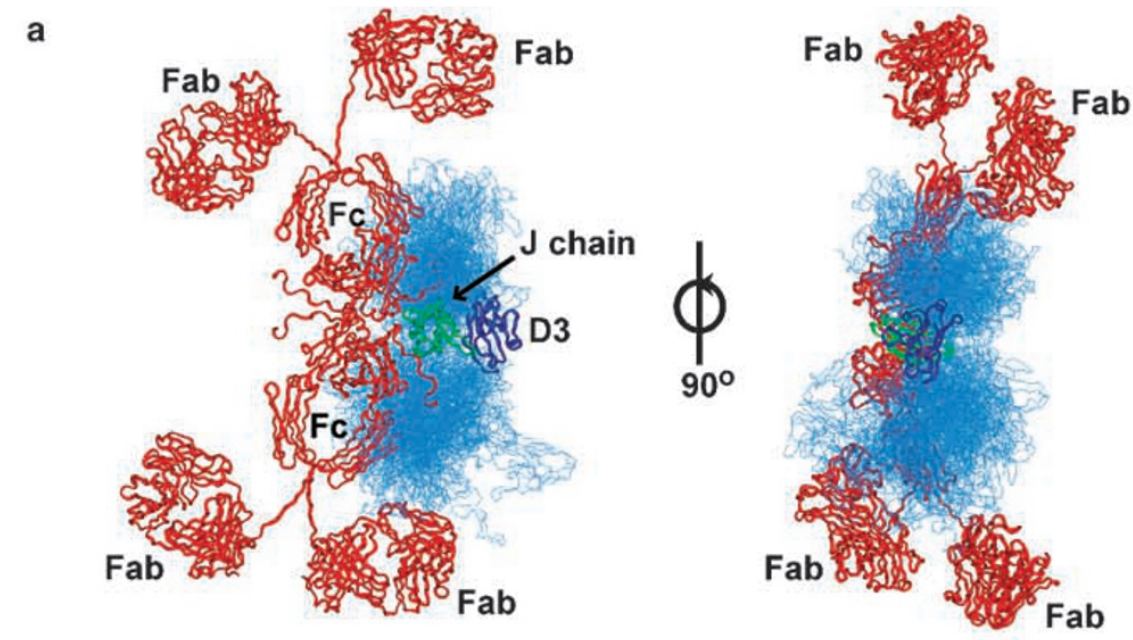

b
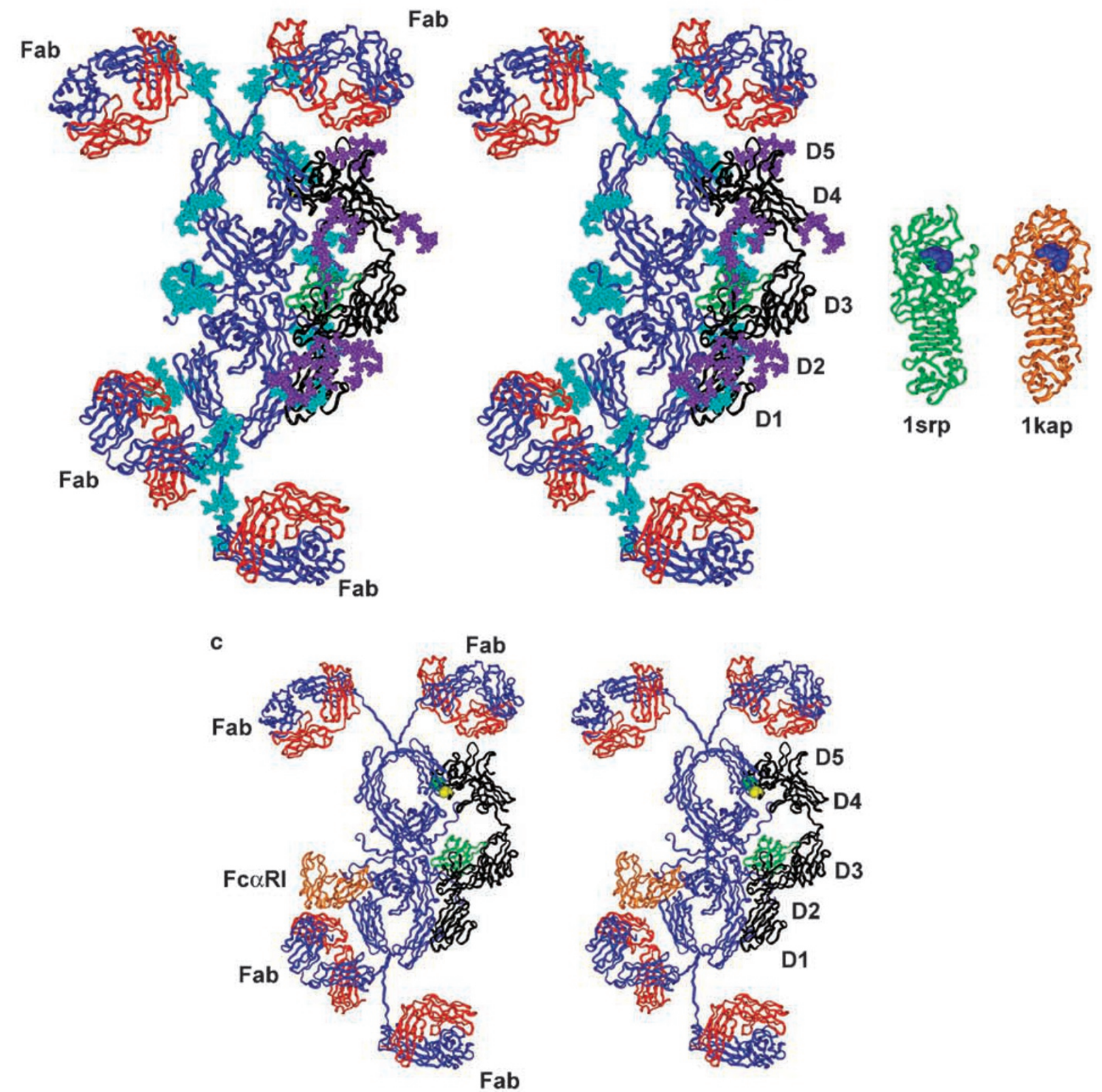
purified using a combination of thiophilic chromatography and jacalinagarose lectin affinity chromatography. ${ }^{14,24,41}$ Dimeric SIgA1 eluted as a single symmetrical peak in Sephacryl S-300 size-exclusion gel filtration. Samples were checked by western blot using an anti-IgA1 Fc-specific antibody. Reducing sodium dodecyl sulfate-polyacrylamide gel electrophoresis showed only the three expected bands for pure SIgA1 corresponding to the IgA1 heavy chain at $62 \mathrm{kDa}$, the light chain and J-chain that co-migrate at around $25 \mathrm{kDa}$ and $\mathrm{SC}$ at around $80 \mathrm{kDa} .{ }^{24}$ The recovery rate for SIgA1 was in excess of $90 \%$. The buffer for X-ray scattering and analytical ultracentrifugation was as previous. ${ }^{14}$ The IgA1, J chain, and SC compositions were as previous. ${ }^{12-14}$ There are $4 \kappa$-light chains, $16 \mathrm{~N}$-linked biantennary and $20 \mathrm{O}$-linked oligosaccharides. From this, SIgA1 has a calculated molecular mass of $424 \mathrm{kDa}$, an unhydrated volume of $539 \mathrm{~nm}^{3}$, a hydrated volume of $712 \mathrm{~nm}^{3}$, an absorption coefficient at $280 \mathrm{~nm}(1 \%, 1 \mathrm{~cm})$ of 12.3 , and a partial specific volume $v^{-}$of $0.723 \mathrm{ml} / \mathrm{g}$. ${ }^{42}$

\section{X-ray and neutron scattering and ultracentrifugation data}

$\mathrm{X}$-ray scattering data were obtained in one beam session at the European Synchrotron Radiation Facility, Grenoble, France on the Beamline ID02 in single-bunch mode. ${ }^{14,43}$ Comparison of the $I(Q)$ data for SIgA1 for a single time frame and that averaged over 10 consecutive frames showed excellent signal-noise ratios with no radiation damage or aggregation effects. Only the first single time frames were used for analyses. Neutron scattering data were obtained in one beam session on Instrument LOQ at the Rutherford Appleton Laboratory, Didcot, UK. ${ }^{14,44}$ Concentrations were 0.30 and $0.60 \mathrm{mg} / \mathrm{ml}$. Data reduction and the Guinier and $P(r)$ analyses were as described previously to yield $R_{\mathrm{G}}$ and $I(0)$ values and to transform the $I(Q)$ data into real space. ${ }^{45}$ The anisotropy ratio $R_{\mathrm{G}} / R_{\mathrm{O}}$ (where $R_{\mathrm{O}}$ is the $R_{\mathrm{G}}$ value of the sphere with the same volume as the dry glycoprotein) permits the elongation of different macromolecules to be compared. The normalization of neutron Guinier $I(0)$ values using sample concentrations $c$ and a standard deuterated polymer gives the molecular mass $=I(0) / c \times 9.10^{5} .{ }^{12}$ For the $P(r)$ analyses, the X-ray $I(Q)$ curve contained 521 data points between $Q$ values of $0.08-2.33 \mathrm{~nm}^{-1}$ (fitted with $D_{\max }$ set as $26 \mathrm{~nm}$ ) and the neutron $I(Q)$ curve contained 66 data points between $Q$ values of $0.13-2.1 \mathrm{~nm}^{-1}$ (fitted with $D_{\max }$ set as $24 \mathrm{~nm}$ ).

Sedimentation equilibrium runs were performed at $20^{\circ} \mathrm{C}$ on a Beckman XL-I instrument equipped with an AnTi50 rotor at three concentrations of $0.20,0.42$, and $0.65 \mathrm{mg} / \mathrm{ml}$ and four rotor speeds of $5,000,8,000,11,000$, and 14,000 r.p.m. Data were fitted to a single-species model. Sedimentation velocity data were acquired at rotor speeds of $10,000,15,000$, and 20,000 r.p.m. at concentrations of $0.15,0.31$, and $0.58 \mathrm{mg} / \mathrm{ml}$. Other details are as described previously. ${ }^{14,45}$ The frictional ratio $f / f_{\mathrm{o}}$ (where $f_{\mathrm{o}}$ is the frictional coefficient of a sphere with a volume equal to that of the hydrated macromolecule) also permits the elongation of different macromolecules to be compared.

\section{Constrained modeling of SIgA1}

The scattering and sedimentation data were subjected to constrained modelling to determine the SIgA1 solution structure. ${ }^{16}$ The constraints were the recently determined dIgA1 solution structure (PDB code $2 \mathrm{qtj})^{14}$ and the 5,000 conformationally randomized SC models that were generated by the unrestrained linker search. ${ }^{13}$ The first cycle of searches fixed the $\mathrm{D} 3$ or $\mathrm{D} 5$ domain of the SC models in 10 distinct locations adjacent to dIgA1 (Figure 6a), then evaluating the resulting SIgA1 models. Searches 1 and 2 were performed with D3 next to the J chain domain on the convex edge of the Fc region in which D3 was aligned with the plane of the Fc region or perpendicular to this. Searches 3 and 4 held D3 in two similar orientations on the other concave edge of the Fc region. Searches 5 and 6 held D3 in two similar orientations on top of the center of the Fc dimeric region. Searches 7-10 were performed with D5 held adjacent to the four $\mathrm{C}_{\mathrm{H}} 2$ domains at the top of the Fc regions. The second cycle of searches randomized the hinge between the $\mathrm{Fab}$ and $\mathrm{Fc}$ regions in the best-fit SIgA1 structure obtained from the first cycle (Figure 5a). ${ }^{12,14}$ Six linker libraries with 5,000 hinge conformations in each were generated using molecular dynamics (DISCOVER module of INSIGHT II, Accelrys) for 25-residue hinge lengths set between 5.5 and $9.0 \mathrm{~nm}$ in $0.5 \mathrm{~nm}$ steps, from each of which 500 conformations were randomly selected. The hinge between the $\mathrm{C}_{\mathrm{H}} 1$ and $\mathrm{C}_{\mathrm{H}} 2$ domains is 220-CPVPSTPPTPSPSTPPT PSPSCCHP-244. ${ }^{12}$ Four identical hinges were used to generate each of the 3,000 SIgA1 models.

Each SIgA1 coordinate model was converted into Debye spheres to calculate X-ray and neutron scattering curves and sedimentation coefficients for comparison with the experimental curves, as previously described. ${ }^{12,14,45}$ The 10 best-fit SIgA1 $\alpha$-carbon coordinate models were deposited in the PDB with the accession code 3chn (Supplementary Figure S1 online).

SUPPLEMENTARY MATERIAL is linked to the online version of the paper at http://www.nature.com/mi

\section{ACKNOWLEDGMENTS}

We thank the Biological and Biotechnology Sciences Research Council and the Wellcome Trust for financial support. We also thank Mr J Gor for excellent assistance with the ultracentrifuge; Dr A Robertson and Dr PW Whitty for assistance with part of the scattering data collection; and $\mathrm{Dr} S$ Finet (ESRF, Grenoble), Dr RK Heenan, and Dr SM King (ISIS, RutherfordAppleton Laboratory) for instrumental support.

\section{DISCLOSURE}

The authors declared no conflict of interest.

(C) 2009 Society for Mucosal Immunology

\section{REFERENCES}

1. Kerr, M.A. The structure and function of human IgA. Biochem. J. 271, 285-296 (1990).

2. Mestecky, J., Moro, I., Kerr, M.A. \& Woof, J.M. Mucosal immunoglobulins. In Mucosal Immunology 3rd edn (Mestecky J.R., Bienenstock J., Lamm M.E., Mayer L., McGhee J.R. \& Strober W., eds) 153-181 (Chapter 9) (Academic Press, San Diego, CA, 2005).

3. Brandtzaeg, P. Induction of secretory immunity and memory at mucosal surfaces. Vaccine 25, 5467-5484 (2007).

4. Crottet, P. \& Corthésy, B. Mapping the interaction between murine IgA and murine secretory component carrying epitope substitutions reveals a role of domains II and III in covalent binding to IgA. J. Biol. Chem. 274, 31456-33146 (1999)

Figure 8 Molecular assembly of the best-fit secretory IgA1 (SIgA1) model. (a) The 34 best-fit models of SlgA1 from search 1 are shown in two orthogonal views that are face-on to (left) and in the plane (right) of the Fc regions. The IgA1 monomers are shown in red, and $\mathrm{J}$ chain is shown in green. The $\alpha$-carbon traces of secretory component (SC) are shown in blue, except for the fixed domain D3, which is shown as a blue ribbon. (b) The best-fit SlgA1 model is shown as a stereo pair in blue (heavy chains), red (light chains), and green (J chain). SC is shown in black with its domains D1-D5 labeled D1 to D5. The $\mathrm{O}$ - and $\mathrm{N}$-linked oligosaccharides in the IgA1 moieties are shown in cyan, whereas the seven $\mathrm{N}$-linked oligosaccharides of SC are shown in purple. Crystal structures for two bacterial zinc-dependent metalloproteases from Pseudomonas aeruginosa and Serratia marcescens (PDB codes 1srp and 1kap) associated with pulmonary or gastrointestinal infections are shown to the right, with the His-Glu residues and zinc at their active sites highlighted in dark blue. (c) The best-fit SIgA1 model is shown relative to the fc $\alpha$ receptor as a stereo pair in the same coloring scheme as in (b). Here, the Fc $\alpha$ receptor is shown in orange, whereas Cys311 in the IgA1 Fc is shown in yellow, and Cys502 in D5 of SC is shown in green. 
5. Lindh, E. Increased resistance of immunoglobulin A dimers to proteolytic degradation after binding of secretory component. J. Immunol. 114, 284-286 (1975).

6. Almogren, A., Senior, B.W., Loomes, L.M. \& Kerr, M.A. Structural and functional consequences of cleavage of human secretory and human serum immunoglobulin $\mathrm{A} 1$ by proteinases from Proteus mirabilis and Neisseria meningitides. Infect. Immun. 71, 3349-3356 (2003).

7. Almogren, A., Senior, B.W. \& Kerr, M.A. A comparison of the binding of secretory component to immunoglobulin A (IgA) in human colostral S-IgA1 and S-IgA2. Immunology 120, 273-280 (2007).

8. Perrier, C., Sprenger, N. \& Corthésy, B. Glycans on secretory component participate in innate protection against mucosal pathogens. J. Biol. Chem. 281, 14280-14287 (2006).

9. Phalipon, A. \& Corthésy, B. Novel functions of the polymeric lg receptor: well beyond transport of immunoglobulins. Trends Immunol. 24, 55-58 (2003).

10. Svehag, S.-E. \& Bloth, B. Ultrastructure of secretory and high-polymer serum immunoglobulin A of human and rabbit origin. Science 168, 847-849 (1970).

11. Bloth, B. \& Svehag, S.-E. Further studies on the ultrastructure of dimeric IgA of human origin. J. Exp. Med. 133, 1035-1042 (1971).

12. Boehm, M.K., Woof, J.M., Kerr, M.A. \& Perkins, S.J. The Fab and Fo fragments of IgA1 exhibit a different arrangement from that in IgG: a study by X-ray and neutron solution scattering and homology modelling. J. Mol. Biol. 286, 1421-1447 (1999).

13. Bonner, A., Perrier, C., Corthésy, B. \& Perkins, S.J. The solution structure of human secretory component and implications for biological function. J. Biol. Chem. 282, 16969-16980 (2007).

14. Bonner, A., Furtado, P.B., Almogren, A., Kerr, M.A. \& Perkins, S.J. Implications of the near-planar solution structure of human myeloma dimeric IgA1 for mucosal immunity and IgA nephropathy. J. Immunol. 180, 1008-1018 (2008).

15. Almogren, A., Furtado, P.B., Sun, Z., Perkins, S.J. \& Kerr, M.A. Biochemical and structural properties of the complex between human immunoglobulin $\mathrm{A} 1$ and human serum albumin by $X$-ray and neutron scattering and analytical ultracentrifugation. J. Mol. Biol. 356, 413-431 (2006).

16. Perkins, S.J., Okemefuna, A.I., Fernando, A.N., Bonner, A., Gillbert, H.E. \& Furtado, P.B. X-ray and neutron scattering data and their constrained molecular modelling. Mol. Cell Biol. 84, 375-423 (2008).

17. Cole, J.L., Lary, J.W., Moody, T.P. \& Laue, T.M. Analytical ultracentrifugation: sedimentation velocity and sedimentation equilibrium. Mol. Cell Biol. 84, 143-211 (2008).

18. Bjork, I. \& Lindh, E. Gross conformation of human secretory immunoglobulin A and its component parts. Eur. J. Biochem. 45, 135-145 (1974).

19. Woof, J.M. \& Kerr, M.A. The function of immunoglobulin A in immunity. J. Pathol. 208, 270-282 (2006).

20. Heremans, J.F. Immunoglobulin A In The Antigens Vol. 2 (Sela, M., ed) 365-522 (Academic Press, New York, 1974).

21. Corthésy, B. Recombinant secretory immunoglobulin A in passive immunotherapy: linking immunology and biotechnology. Curr. Pharm. Biotechnol. 4, 51-67 (2003).

22. Mestecky, J. \& McGhee, J.R. Immunoglobulin A (IgA): molecular and cellular interactions involved in IgA biosynthesis and immune response. Adv. Immunol. 40, 153-245 (1987).

23. Fallgreen-Gebauer, E. et al. The covalent linkage of secretory component to IgA: structure of slgA. Biol. Chem. Hoppe Seyler. 374, 1023-1028 (1993).

24. Almogren, A. \& Kerr, M.A. Irreversible aggregation of the Fc fragment derived from polymeric but not monomeric serum IgA1 -implications in IgA-mediated disease. Mol. Immunol. 45, 87-94 (2008).

25. Herr, A.B., Ballister, E.R. \& Bjorkman, P.J. Insights into IgA-mediated immune responses from the crystal structures of human FcaRl and its complex with IgA1-Fc. Nature 423, 614-620 (2003).
26. Perkins, S.J., Nealis, A.S., Sutton, B.J. \& Feinstein, A. Solution structure of human and mouse immunoglobulin M by synchrotron X-ray scattering and molecular graphics modelling: a possible mechanism for complement activation. J. Mol. Biol. 221, 1345-1366 (1991).

27. Deisenhofer, J. Crystallographic refinement and atomic models of a human Fc fragment and its complex with fragment B of protein A from Staphylococcus aureus at 2.9 and $2.8 \AA$ resolution. Biochemistry 20, 2361-2370 (1981).

28. Monteiro, R.C. \& van de Winkel, J.G.J. IgA Fc receptors. Annu. Rev. Immunol. 21, 177-204 (2003).

29. Woof, J.M., van Egmond, M. \& Kerr, M.A. Fc receptors. In Mucosal Immunology 3rd edn (Mestecky, J.R., Bienenstock, J., Lamm, M.E., Mayer, L., McGhee, J.R., \& Strober, W., eds) 251-266 (Academic Press, San Diego, CA, 2005).

30. Mazengera, R.L. \& Kerr, M.A. The specificity of the IgA receptor purified from human neutrophils. Biochem. J. 272, 159-165 (1990).

31. Herr, A.B., White, C.L., Milburn, C., Wu, C. \& Bjorkman, P.J. Bivalent binding of IgA1 to FcaRl suggests a mechanism for cytokine activation of IgA phagocytosis. J. Mol. Biol. 327, 645-657 (2003).

32. Pleass, R.J., Dunlop, J.I., Anderson, C.M. \& Woof, J.M. Identification of residues in the $\mathrm{CH} 2 / \mathrm{CH} 3$ domain interface of IgA essential for interaction with the human Fc $\alpha$ receptor (Fc $\alpha$ RI) CD89. J. Biol. Chem. 274, 23508-23514 (1999).

33. Brandtzaeg, P. \& Prydz, H. Direct evidence for an integrated function of $J$ chain and secretory component in epithelial transport of immunoglobulins. Nature 311, 71-73 (1984).

34. Johansen, F.-E., Braathen, R. \& Brandzaeg, P. The J chain is essential for polymeric Ig receptor-mediated epithelial transport of IgA. J. Immunol. 167, 5185-5192 (2001).

35. Brandtzaeg, P. Mucosal and glandular distribution of immunoglobulin components: differential localization of free and bound SC in secretory epithelial cells. J. Immunol. 112, 1553-1559 (1974).

36. Bakos, M.-A., Kurosky, A. \& Goldblum, R.M. Characterization of a critical binding site for human polymeric Ig on secretory component. J. Immunol. 147, 3419-3426 (1991).

37. Hamburger, A.E., West, A.P. \& Bjorkman, P.J. Crystal structure of a polymeric immunoglobulin binding fragment of the human polymeric immunoglobulin receptor. Structure 12, 1925-1935 (2004).

38. Norderhaug, I.N, Johansen, F.-E., Krajci, P. \& Brandtzaeg, P. Domain deletions in the human polymeric Ig receptor disclose differences between its dimeric IgA and pentameric IgM interaction. Eur. J. Immunol. 29, 3401-3409 (1999).

39. Beale, D. Differences in fragmentation between bound and unbound bovine secretory component suggest a model for its interaction with polymeric immunoglobulin. Biochem. J. 229, 759-763 (1985).

40. Crottet, P. \& Corthésy, B. Secretory component delays the conversion of secretory IgA into antigen-binding competent $F\left(a b^{\prime}\right) 2$ : a possible implication for mucosal defense. J. Immunol. 161, 5445-5453 (1998).

41. Sun, Z., Almogren, A., Furtado, P.B., Chowdhury, B., Kerr, M.A. \& Perkins, S.J. Semi-extended solution structure of human myeloma immunoglobulin D determined by constrained X-ray scattering. J. Mol. Biol. 353, 155-173 (2005).

42. Perkins, S.J. Protein volumes and hydration effects: the calculation of partial specific volumes, neutron scattering matchpoints and $280 \mathrm{~nm}$ absorption coefficients for proteins and glycoproteins from amino acid sequences. Eur. J. Biochem. 157, 169-180 (1986).

43. Narayanan, T., Diat, O. \& Bosecke, P. SAXS and USAXS on the high brilliance beamline at the ESRF. Nucl. Instrum. Methods Phys. Res. A 467-468, 1005-1009 (2001).

44. Heenan, R.K., Penfold, J. \& King, S.M. SANS at pulsed neutron sources: present and future prospects. J. Appl. Crystallogr. 30, 1140-1147 (1997).

45. Furtado, P.B. et al. Solution structure determination of human IgA2 by $X$-ray and neutron scattering and analytical ultracentrifugation and constrained modelling: a comparison with human IgA1. J. Mol. Biol. 338, 921-941 (2004). 